This item was submitted to Loughborough's Research Repository by the author.

Items in Figshare are protected by copyright, with all rights reserved, unless otherwise indicated.

\title{
Theoretical model of Rayleigh wave interaction with stripes of viscous liquids
}

PLEASE CITE THE PUBLISHED VERSION

PUBLISHER

(C) IEEE

VERSION

VoR (Version of Record)

LICENCE

CC BY-NC-ND 4.0

\section{REPOSITORY RECORD}

Krylov, Victor V., M.I. Newton, J.A. Cowen, M.K. Banejee, and G. McHale. 2010. "Theoretical Model of Rayleigh Wave Interaction with Stripes of Viscous Liquids". figshare. https://hdl.handle.net/2134/6158. 
This item was submitted to Loughborough's Institutional Repository (https://dspace.lboro.ac.uk/) by the author and is made available under the following Creative Commons Licence conditions.

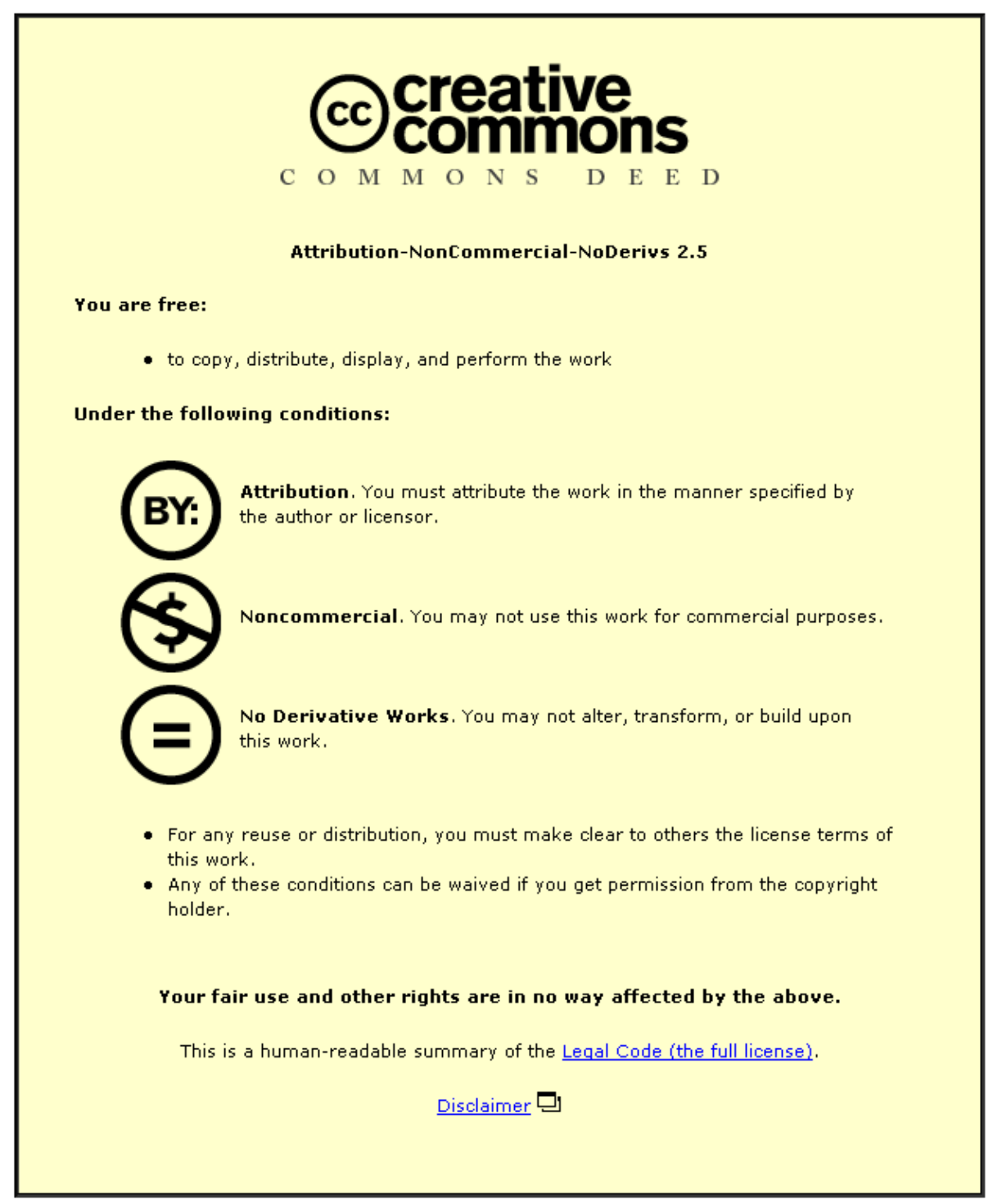

For the full text of this licence, please go to: http://creativecommons.org/licenses/by-nc-nd/2.5/ 


\title{
Theoretical Model of Rayleigh Wave Interaction with Stripes of Viscous Liquids
}

\author{
V.V. Krylov", M.I. Newton", J.A. Cowen", M.K. Banerjee* and G. M'Hale* \\ "Department of Civil and Structural Engineering \\ The Nottingham Trent University \\ Burton Street, Nottingham NGl 4BU, UK \\ "Department of Chemistry and Physics \\ The Nottingham Trent University \\ Clifton Lane, Nottingham NG1 1 8NS, UK
}

\begin{abstract}
The problem of Rayleigh surface wave transmission and reflection by stripes of viscous liquid is considered using a Maxwellian model of viscosity characterised by a single relaxation time. The aim is to interpret experiments on surface acoustic wave monitoring of the wetting of highenergy solid surfaces (G. McHale et al., Faraday. Discuss., 1997, 107, 27-38). These experiments demonstrated a strong oscillatory behaviour of both transmission and reflection coefficients as the area occupied by the liquid increased with time. The variable thickness of the liquid is taken into account and analytical results and numerical calculations are presented.
\end{abstract}

\section{INTRODUCTION}

Surface acoustic waves (SAW's) are one of a broad class of acoustic techniques that have been applied to the study of physical changes at the solid-liquid interface. Other examples include shear horizontally polarised surface acoustic waves, acoustic plate modes, Love waves and quartz crystal microbalances. Most studies of liquids using these acoustic techniques have been limited to fixed pools of liquid in contact with a device surface. Almost no studies have been published in which the liquid is localised and dynamically evolving. One exception is the work of $\mathbf{M}^{c} \mathrm{Hale}$ et al [1,2] which used pulses of high frequency (170 MHz) Rayleigh surface acoustic waves and dynamically evolving stripes of a viscous oil. These experiments showed that resonant reflections of SAW's are observed for particular geometrical parameters of the stripe and that the transmitted SAW signal does not decrease steadily as the stripe spreads, but shows specific significant attenuation. In this paper, we present a theoretical model to account for the changes observed in the reflection and transmission co-efficients of pulses of high frequency Rayleigh surface acoustic waves as a stripe of viscous fluid wets the substrate surface.

\section{EXPERIMENTAL SYSTEM}

The experimental system used to investigate the interaction of Rayleigh waves with wetting oils is shown schematically in Fig. 1.

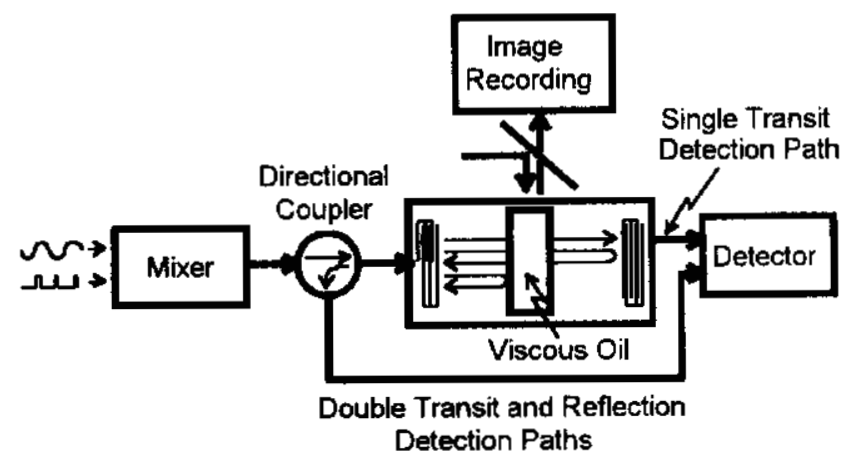

Figure 1 Experimental arrangement.

The surface acoustic wave of frequency $170 \mathrm{MHz}$ (wavelength $\lambda \approx 22 \mu \mathrm{m}$ ) is generated by a set of interdigital transducers operated in a pulsed mode. The SAW travels along the unloaded surface of the substrate until it reaches the region of the device which is loaded with a parallel stripe of a viscous oil, poly(dimethyl)siloxane, with a viscosity between $10000 \mathrm{cS}$ and $100000 \mathrm{cS}$. The cross-section of the liquid is a spherical cap shape characterised by a contact width, $d$, a height, $h$, and a spherical radius, $r$. A reflection of the SAW occurs from the oil and this is detected using the original transducer. Another 
signal, the single transit, passes along the substrateliquid interface and is detected by a second set of transducers. The time of transit allows the reflection signal to be unambiguously identified as a reflection from the liquid loaded region of the device. A simple interferometry arrangement using sodium light allows the geometric profile of the oil to be recorded onto videotape during the course of the spreading. It is therefore possible to correlate changes in the geometry of the oil with the reflection and transmission of the surface acoustic wave.

\section{THEORY}

\section{SAW Reflection}

To calculate the reflection of surface acoustic waves from the stripes of viscous liquid we model a stripe as one having trapezoidal shape and apply the theory of Rayleigh wave scattering by small surface inhomogeneities [3] to the scattering by front and rear slopes of the stripe. According to this theory, the acoustic displacements $u_{m}$ in the slope area of the device substrate modified by the presence of viscous Maxwellian liquid satisfy the following integrodifferential equation at the solid/liquid interface $z=0$

$$
\begin{aligned}
& u_{m}(r)=u_{m}^{o}(r)+ \\
& \int_{-\infty}^{\infty}\left[f_{1}^{*}\left(u_{i}\left(r^{\prime}\right)\right) G_{1 m}\left(r, r^{\prime}\right)+f_{2}^{*}\left(u_{i}\left(r^{\prime}\right)\right) G_{2 m}\left(r, r^{\prime}\right)\right] d x
\end{aligned}
$$

Here $u_{m}{ }^{\circ}$ is the displacement vector of an incident surface wave, $f_{1}^{*}$ and $f_{2}^{*}$ are the differential operators describing modification of the boundary conditions on the solid surface due to the presence of liquid slope [4], and $G_{1 m}$ and $G_{2 m}$ are the corresponding components of the elasto-dynamic Green's tensor for elastic half space.

The viscous nature of the stripe of fluid is taken into account by using the Maxwell model, which introduces a single relaxation time, $\tau=\eta / \mu$, where $\eta$ is the viscosity and $\mu$ is the high frequency shear modulus. The viscoelastic fluid has both elastic and damping components to the rate of strain, $\dot{\gamma}$, which is described by

$$
\dot{\gamma}=\dot{\gamma}_{E}+\dot{\gamma}_{V}=\frac{\dot{\sigma}}{\mu}+\frac{\sigma}{\eta}
$$

where $\sigma$ is the shear stress. The relaxation time factor, gives two limits, i) a Newtonian liquid when $\omega \tau \rightarrow 0$, and ii) an amorphous solid when $\omega \tau \rightarrow \infty$.

Solving Eq. (1) in the first Born approximation and using the definition of the reflection coefficient as $u_{m}^{b s c} / u_{m}{ }^{o}$, where $u_{m}^{b s c}$ is a backscattered acoustic field, result in the analytical expressions for the reflection coefficients for the front slope of the stripe, $R_{l}$, and for its rear slope, $R_{2}=-R_{1}$ (which are omitted here for briefness). After that, the resulting coefficient of the SAW reflection from the whole stripe as a function of its width $d$ can be modelled as

$$
R=R_{1}\left[1-e^{2 i k 1(d) d}+H\left(c_{t}-c^{(2)}(d)\right) D e^{2 i k 2(d) d}\right]
$$

Here $k l(d)$ is the stripe-width dependent wave number of a lowest order SAW mode describing its dispersion (we recall that the maximal stripe thickness $h$ decreases with the increase of $d$, so that the cross-sectional areas of slopes and of the stripe as a whole remain constant), $H\left(c_{r}-c^{(2)}(d)\right)$ is a Heaviside step function describing the appearance of a second SAW mode with a wavenumber $k 2(d)$ when its phase velocity $c^{(2)}(d)$ becomes smaller than the velocity of shear waves in the substrate, i.e. for relatively thick fluids, and $D$ is the empirical 'fitting' parameter describing the amplitude of generated second mode. As will be discussed below, the inclusion into Eq. (3) of the effects of dispersion and second mode excitation is paramount for understanding the experimental results on SAW reflection from liquid stripes.

\section{SAW Transmission}

To calculate the transmission coefficient of surface acoustic waves we consider the acoustic losses due to the shear motion induced in the stripe of liquid on the device surface using a model earlier applied for Newtonian liquids on thickness shear mode resonators [5]. Our model assumes that,

1. the motion of the substrate is damped simple harmonic motion;

2. the main damping is due to shear motion induced in the fluid overlayer of thickness $t_{f}$,

3. the substrate depth in motion is $\xi \lambda_{\text {SAw }}$ with $\xi \sim 1$;

4. the fluid is visco-elastic and obeys a Maxwell model. 
For a damped harmonic oscillator the equation of motion is,

$$
F=\frac{1}{2} \rho_{s} t_{s}\left(\ddot{q}+\gamma_{d} \dot{q}+\omega_{o}^{2} q\right)-F_{f}
$$

where $q=A_{o} e^{i \omega t}$ is the (in-plane) displacement of the harmonic oscillator at time $t, F_{f}$ is the force exerted by the fluid on the substrate per unit surface area, $\gamma_{d}$ and $\omega_{o}$ are the damping and resonant frequency of the unloaded substrate, respectively, $\rho_{s}$ is the density of the substrate and $t_{s}$ is the thickness of oscillating substrate. For a surface acoustic wave the substrate thickness is modelled by a characteristic depth of penetration of $t_{s}=\xi \lambda_{\text {SAW }}$.

The fluid velocity, $v_{f}$, obeys the Navier-Stokes equation. Solving this equation subject to the boundary conditions at the solid/liquid interface $z=0$ and at the free surface of the liquid, gives the following expression for the force per unit area,

$$
F_{f}=\frac{\beta v_{o}}{1+i \omega \tau}
$$

where

$$
\beta=(\eta \sqrt{2 i} / \alpha)\left(1-i k^{2} \alpha^{2} / 2\right) \tanh \left[\sqrt{2 i} t_{f} / \alpha\right]
$$

and

$$
\alpha=\frac{\delta}{\sqrt{1+i \omega \tau-i \delta^{2} k^{2} / 2}}
$$

In these expressions $k=2 \pi / \lambda_{\mathrm{SAW}}$ and $\delta=\sqrt{ }(2 \eta / \omega \rho)$ is the penetration depth into the liquid. Since the additional damping arising from the fluid is proportional to the substrate velocity, $v_{o}, \mathrm{Eq}$. (4) enables the acoustic loss to be calculated (in $\mathrm{dB}$ ) as

$$
L \approx-20 \log _{10} e \frac{d}{\rho_{s} t_{s} v_{S A W}} \operatorname{Real}\left[\frac{\beta}{1+i \omega \tau}\right]
$$

Similar solutions can be obtained for both thickness shear mode and shear horizontal surface acoustic wave modes. These equations can be examined in various analytical limits and do reduce to known solutions $[6,7]$.

\section{RESULTS AND DISCUSSION}

Analysis of the reflection coefficient, Eq. (3) shows that it decreases with aperiodic oscillations as the liquid spreads. Note that the quasi-period of these oscillations is larger for smaller values of stripe width $d$ (larger values of thickness $h$ ) which is due to the effect of increasing surface wave dispersion. The effect of emerging second SAW mode for small values of $d$ results in diminished amplitudes of the reflection coefficient and its more frequent oscillations.

The results of calculation of the reflection coefficient for the liquid stripe being effectively a solid are shown in Fig. 2 together with the corresponding experimental data. It is seen that the qualitative agreement between the theory and the experiment is quite reasonable, thus indicating the correct understanding of the physical processes involved.

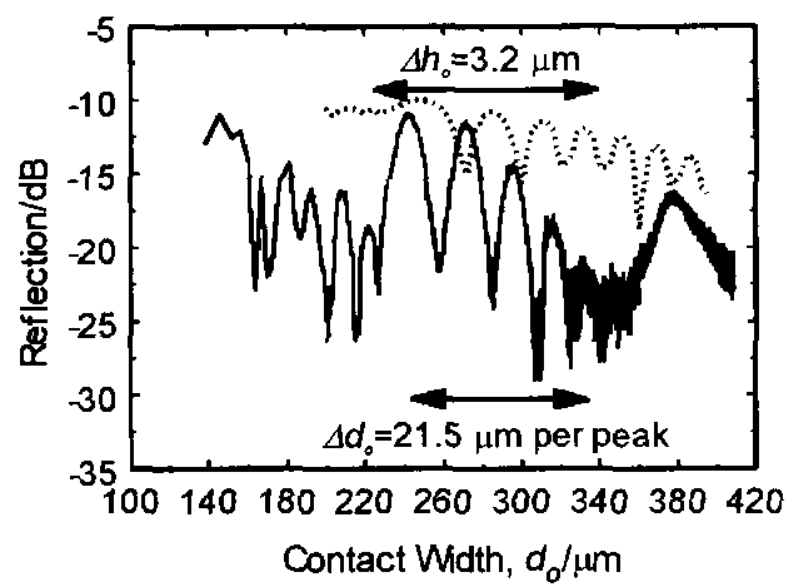

Figure 2 Resonant reflections occur from the stripe.

Regarding the transmission coefficient, Eq. (8), one can see that a significant change in character occurs as the relaxation time changes. In the limit of $\omega \tau \rightarrow 0$ both the frequency shift and acoustic losses saturate once the fluid depth exceeds a few penetration depths. However, in the opposite limit of $\omega \tau \rightarrow \infty$ the acoustic losses show a regular sequence of sharp peaks with a slower overall modulation of the envelope of these peaks. This change with relaxation time represents a change in fluid character from liquid to effectively solid. It is then possible for shear wave resonances to occur when the fluid thickness matches the condition,

$$
t_{f}=n \lambda_{\text {shear }} / 4
$$


where $n=1,3,5, \ldots$. Typically, the double transit signal shows that as the oil spreads steadily across the surface the attenuation increases, with very large attenuation resonances being evident as shown in Fig. 3 (this data is from a different experiment to Fig. 2). These transmission coefficient resonances have a characteristic asymmetric shape. The changes in the single transit signal precisely mirror those of the double transit signal as would be expected. Data from a range of experiments can all be fitted using the relaxation rates obtained from the viscosity and a high frequency shear modulus of $\mu=(1.5 \pm 0.1) \mathrm{GNm}^{-2}$.

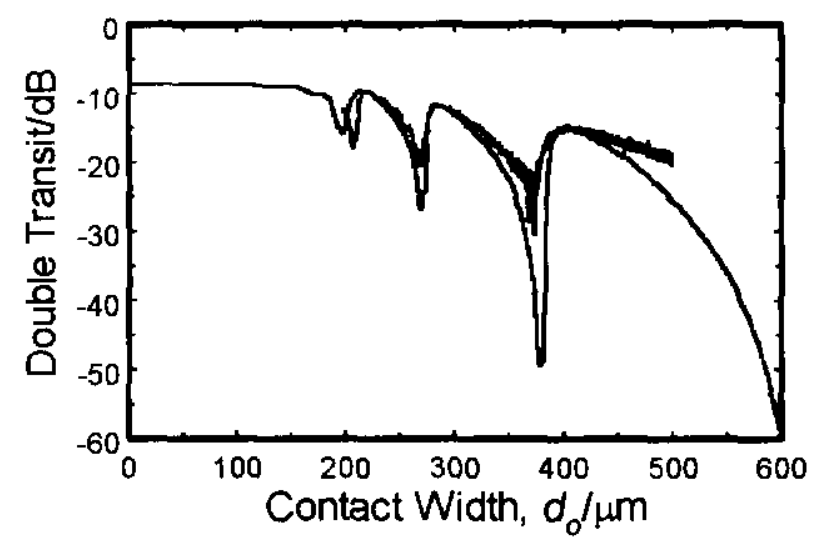

Figure 3 Transit signal and theoretical fit.

\section{CONCLUSION}

The model developed is able to explain the main features observed in the Rayleigh surface wave reflection and transmission coefficients. It also suggests that acoustically we have a creeping amorphous solid rather than a liquid. The high level of detail possible in the experiments, suggests that use of surface acoustic waves for investigating dynamically wetting liquids can clarify the mechanisms of acoustic wave-liquid interfacial interactions; such interactions can be used for acoustic testing of small amounts of liquid. Moreover, the realisation that the large relaxation time limit corresponds to a creeping solid and that the shape of small amounts of oil has a high level of symmetry, also provides a simple test problem for the interaction of SAW's with spatial surface inhomogeneities.

\section{REFERENCES}

[1] G. McHale, M.I. Newton, M.K. Banerjee and S.M. Rowan, "Interaction of surface acoustic waves with viscous liquids", Faraday Discuss., vol 107, pp 15-27, 1997.

[2] M.I. Newton, G. McHale and M.K. Banerjee, "Reflection of surface acoustic waves by localized wetting liquids", Appl. Phys.Lett., vol 71, pp 3785-3786, 1997.

[3] S.V. Biryukov, Yu.V. Gulyaev, V.V. Krylov, and V.P. Plessky, "Surface Acoustic Waves in Inhomogeneous Media", Springer, Berlin, Heidelberg, 1995.

[4] V.V. Krylov, "Surface properties of solids and surface acoustic waves: Application to chemical sensors and layer characterisation", Applied Physics, vol A61, pp229-236, 1995.

[5] M. Rodahl and B. Kasemo, "On the measurement of thin liquid overlayers with the quartz-crystal microbalance", Sensors and Actuators, vol A54, pp 448-456, 1996.

[6] G. McHale, M.K. Banerjee, M.I. Newton and V.V. Krylov, "Surface acoustic wave resonances in the spreading of viscous fluids", submitted to Phys. Rev B, 1998.

[7] A.J. Ricco and S.J. Martin, "Acoustic-wave viscosity sensor", Appl. Phys. Lett., vol 50, pp 1474-1476, 1987.

\section{ACKNOWLEDGEMENT}

The authors would like to acknowledge financial support from EPSRC (Grant GR/L82090). 